Annuaire suisse de politique de développement

27-2 | 2008

Migration et développement: un mariage arrangé

\title{
Les partenariats migratoires, une nouvelle approche de la Suisse?
}

Therese Liechti et Monica Budowski

\section{(2) OpenEdition}

Édition électronique

URL : http://journals.openedition.org/aspd/647

DOI : 10.4000/aspd.647

ISSN : 1663-9669

Éditeur

Institut de hautes études internationales et du développement

\section{Édition imprimée}

Date de publication : 1 décembre 2008

Pagination : 213-219

ISBN : 978-2-940415-07-6

ISSN : 1660-5934

\section{Référence électronique}

Therese Liechti et Monica Budowski, « Les partenariats migratoires, une nouvelle approche de la Suisse? », Annuaire suisse de politique de développement [En ligne], 27-2 | 2008, mis en ligne le 01 octobre 2010, consulté le 08 septembre 2020. URL : http://journals.openedition.org/aspd/647 ; DOI : https://doi.org/10.4000/aspd.647 


\title{
Les partenariats migratoires, une nouvelle approche de la Suisse?
}

\author{
Therese Liechti et Monica Budowski*
}

\section{Introduction}

La politique migratoire suisse a changé de perspective ces dernières années. Alors qu'il incombait jusqu'ici à l'Etat de veiller à la sécurité de ses ressortissants - faisant ainsi de la migration un domaine réservé de la nation immédiatement concernée -, ce nouvel éclairage encourage la coopération internationale et transforme cette problématique en domaine partagé. La migration n'est plus considérée uniquement comme une source de dangers, et son potentiel pour la coopération est mis au premier plan. En Suisse, la migration est depuis les années 1960 un sujet sensible qui a entraîné des modifications successives des lois sur l'asile et sur les étrangers, dont la dernière a été votée en 2006. La législation helvétique a officialisé alors la notion de partenariats migratoires (PM), afin d'améliorer la coopération avec les pays d'où proviennent les immigrés. C'est ainsi que la Suisse possède désormais un instrument juridique incluant politique étrangère, politique intérieure et politique de développement. L'objectif du présent article est de décrire d'une part la genèse de cette disposition légale et, d'autre part, les objectifs et les contenus de ces nouveaux partenariats.

\section{La genèse de l'article 100 LEtr sur les partenariats migratoires}

La Suisse a instauré ces dernières années, dans le domaine des migrations, divers instruments politiques tels que l'aide au retour ou l'aide structurelle. Mais le Conseil fédéral suisse a constaté en 2003 que sa marge de manœuvre pour l'organisation de la politique migratoire - dont en particulier ce qui concerne le retour - était limitée par des contraintes de politique intérieure et extérieure, surtout au niveau de sa coopération avec les pays d'origine des demandeurs d'asile déboutés ${ }^{1}$.

Cette problématique a suscité la même année le dépôt d'une initiative cantonale dont l'objectif était d'obliger le Conseil fédéral à supprimer toute aide aux Etats qui adoptent une attitude peu coopérative lors du renvoi de leurs ressortissants ${ }^{2}$. Le parlement a réservé un accueil favorable à cette idée, laquelle s'est concrétisée

* Therese Liechti, lic. phil., chaire de travail social et de politiques sociales, Université de Fribourg. Monica Budowski, Prof. Dr., chaire de travail social et de politiques sociales, Université de Fribourg.

1 Office fédéral des réfugiés, Institution d'un groupe de travail pour de nouveaux progrès dans le domaine de la migration et des retours, communiqué de presse, 16 avril 2003, <http://www.ejpd. admin.ch/ejpd/fr/home/dokumentation/mi/2003/2003-04-160.html> (consulté le 16 juillet 2007).

2 Initiative cantonale 03.304, Loi sur l'asile: modification partielle, <http://www.parlament.ch/F/Suche/ Pages/geschaefte.aspx?gesch_id=20030304> (consulté le 4 mars 2008). 
par l'article 77 de la Loi sur l'asile (LAsi), que le Conseil national a avalisé lors de sa session extraordinaire de mai 2004.

En 2003 toujours, le Conseil fédéral a mis sur pied un groupe de travail dont la mission était de rédiger un rapport passant en revue les instruments de politique extérieure susceptibles de contribuer à une stratégie politique globale en matière de migration ${ }^{3}$.

Ce rapport final du groupe de travail interdépartemental Migration (IDAG Migration) évoque pour la première fois le principe de partenariats migratoires. Il observe notamment que la Suisse devrait à terme se donner pour objectif d'établir des partenariats dans ce domaine avec les pays de provenance et de transit de personnes migrantes. Ces partenariats viseront à concilier les intérêts en présence face aux problèmes posés par l'émigration, l'immigration et le retour ${ }^{4}$. Sur ce, l'Office fédéral des migrations (ODM) et le Département fédéral des affaires étrangères (DFAE) ont élaboré conjointement un projet de texte intégré finalement dans la nouvelle Loi fédérale sur les étrangers (art. 100 LEtr), entrée en vigueur le $1^{\text {er }}$ janvier 2008; cette disposition remplace l'article 77 LAsi. Parallèlement, la Suisse s'est mise à participer plus activement au dialogue international sur les problèmes de migration. Un exemple de succès à cet égard est l'Initiative de Berne, qui a conduit 120 pays à adopter un «Agenda international pour la gestion des migrations » (AIGM); son but est de renforcer la coopération entre Etats sur les questions de migration ${ }^{5}$.

\section{Figure 1: Facteurs ayant conduit à institutionnaliser les partenariats migratoires}

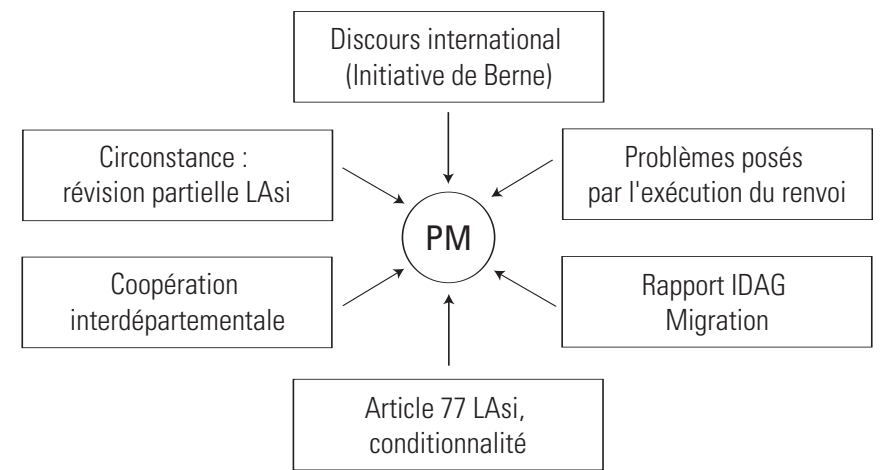

Source: d'après T. Liechti.

3 Interdepartementale Arbeitsgruppe «Aussenpolitik im Migrations- und Rückkehrbereich» (IDAG Migration), Schlussbericht der IDAG Migration, Bern, 2004. Il existe un résumé en français de ce rapport: $<$ http://www.bfm.admin.ch/etc/medialib/data/migration/rueckkehr.Par.0003.File.tmp/040623e_zus-f. $\mathrm{pdf}>$.

4 Ibid., p. 13.

5 C. De Matos et D. Efionayi-Mäder, «Begriffsentstehung, Definition und Ausrichtung von Migrationspartnerschaften in der Schweiz», in Organisation internationale pour les migrations (dir.), Enquête sur les partenariats migratoires: présentation du concept dans les contextes international et suisse, Berne, Organisation internationale pour les migrations (OIM), 2007, p. 36. La version française de cet article peut être commandée auprès de l'OIM Berne. 
Le principe des partenariats migratoires s'est finalement concrétisé dans la nouvelle Loi fédérale sur les étrangers (LEtr) (voir encadré).

\section{Art. 100 Conventions internationales}

${ }^{1}$ Le Conseil fédéral encourage les partenariats bilatéraux et multilatéraux avec d'autres Etats dans le domaine des migrations. II peut conclure des accords visant à renforcer la coopération dans le domaine migratoire et à lutter contre la migration illégale et ses conséquences négatives.

${ }^{2}$ Le Conseil fédéral peut conclure avec des Etats étrangers ou des organisations internationales des conventions sur:

a. les visas et les contrôles à la frontière;

b. la réadmission et le transit des personnes qui se trouvent en situation irrégulière en Suisse;

c. le transit de personnes sous escorte policière, dans le cadre des accords de transit et de réadmission, y compris le statut juridique des agents d'escorte des Etats parties;

d. le délai d'octroi de l'autorisation d'établissement;

e. la formation et le perfectionnement professionnels;

f. le recrutement de travailleurs étrangers;

g. les prestations de services transfrontaliers;

h. le statut juridique des personnes mentionnées à l'art. 98, al. 2.

${ }^{3}$ Dans le cadre de conventions de réadmission et de transit, le Conseil fédéral peut, dans les limites de ses compétences, accorder ou retirer le bénéfice de prestations ou d'avantages. II tient compte des obligations de droit international de la Suisse ainsi que de l'ensemble des relations existant entre la Suisse et l'Etat concerné.

${ }^{4}$ Les départements compétents peuvent conclure avec des autorités étrangères ou des organisations internationales des arrangements sur l'application technique des conventions visées à l'al. 1.

Source: Assemblée fédérale de la Confédération suisse, Loi fédérale sur les étrangers, 2005, <http://www.admin.ch/ ch/f/ff/2005/6885.pdf> (consulté le 29 août 2007).

Les quelques documents faisant état de partenariats migratoires montrent que la LEtr autorise diverses interprétations et applications des critères énumérés cidessus. Cette pluralité de significations possibles a fait l'objet d'une enquête basée sur 16 entretiens menés avec divers responsables ${ }^{6}$ de l'administration fédérale suisse concernés par la problématique des partenariats migratoires ${ }^{7}$. Voici les points de vue des experts interrogés sur les contenus et les objectifs possibles de ces partenariats.

\section{Les partenariats migratoires}

Les partenariats migratoires sont toujours en voie de structuration et s'inscrivent dans un processus dynamique. Objectifs concrets, contenus, procédures à suivre en sont au stade des négociations et/ou d'une première application. Il ressort des

6 T. Liechti, Migrationspartnerschaften: ein neuer Ansatz der schweizerischen Migrationspolitik?, Lizentiatsarbeit, Fribourg, Departement Sozialarbeit und Sozialpolitik, Universität Fribourg, 2007.

7 Organisation internationale pour les migrations, Enquête sur les partenariats migratoires, Berne, Organisation internationale pour les migrations (OIM), 2007. 
interviews menées avec différents spécialistes de l'administration fédérale helvétique que ces partenariats ont les caractéristiques suivantes (voir figure 2):

$1^{\circ}$ le mode de coopération avec les pays de provenance et de transit;

$2^{\circ}$ l'idée de créer un instrument destiné à gérer efficacement la problématique des migrations;

$3^{\circ}$ la collaboration interdépartementale, condition préalable d'une coopération internationale.

Figure 2: Caractéristiques des partenariats migratoires

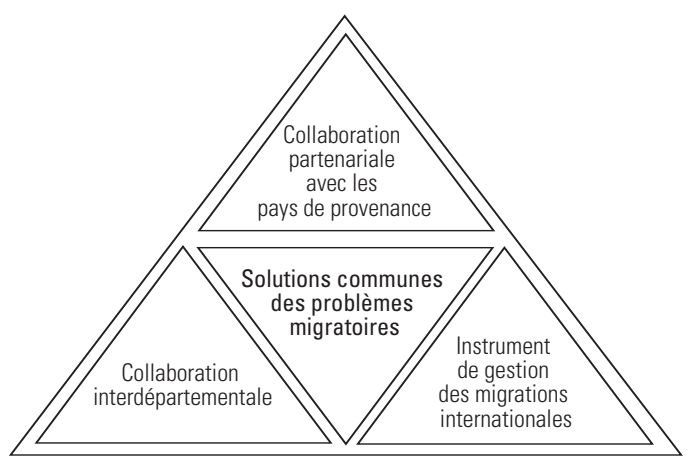

Source: T. Liechti, Migrationspartnerschaften: ein neuer Ansatz der schweizerischen Migrationspolitik?, Lizentiatsarbeit, Fribourg, Departement Sozialarbeit und Sozialpolitik, Universität Fribourg, 2007, p. 97.

\section{Le mode de coopération avec les pays de provenance et de transit}

L'idée qui sous-tend les partenariats migratoires est de trouver avec les pays partenaires, grâce à un dialogue où chaque partie est égale en droits, des solutions à des questions propres à la migration, le but étant de créer des situations satisfaisantes pour toutes les parties en présence (situations win-win). Cette attitude ne doit pas masquer le fait que chaque partenaire s'efforcera de faire prévaloir ses propres intérêts. La Suisse s'est rendu compte, dans ce contexte, qu'elle ne dispose pas de moyens de pression suffisants pour imposer son point de vue et défendre unilatéralement ses intérêts. Elle est donc tributaire de la volonté de coopérer des pays partenaires. Mais il faut avoir déjà établi des relations substantielles avec le pays considéré avant de pouvoir envisager un partenariat migratoire. Par ailleurs, le pays en question doit posséder des structures administratives à peu près fiables et une situation intérieure suffisamment stable. Du côté de la Suisse, une question importante - mis à part les intérêts supradépartementaux en matière de politique migratoire - est de régler le financement des dispositions à prendre. Il s'agit de déterminer quel département pourra consacrer des moyens à l'établissement de partenariats migratoires.

\section{L'instrument de gestion des migrations internationales}

La question qui se pose ensuite est de savoir comment gérer quelle migration. Dans le discours sur la migration, on fait souvent la distinction entre migration voulue et migration non voulue. Dans l'optique des pays d'accueil, on souhaite 
avant tout voir affluer une main-d'œuvre hautement qualifiée, tandis que l'on qualifie d'indésirables les personnes sans qualifications particulières dont il y aurait lieu de craindre qu'elles soient une charge pour l'Etat social ou qu'elles échappent, par un séjour illégal, au contrôle des pouvoirs publics. Les catégories «désirable» et «indésirable» constituent ainsi les catégories clés qui contribuent de manière décisive à la question de savoir si l'immigration sera considérée comme une opportunité ou un risque, et comment le pays d'accueil sera censé gérer le phénomène ${ }^{8}$. Il en résulte toutes sortes d'objectifs et de contenus possibles pour les partenariats migratoires. On observe le souhait manifeste d'un contrôle plus serré, d'une gestion mieux dirigée des migrations. Cela implique des possibilités concrètes de retour pour les demandeurs d'asile déboutés, la prévention de la migration irrégulière ainsi qu'une gestion améliorée de cette problématique dans le pays partenaire (capacity building). Les partenariats migratoires ont pour caractéristique de ne pas se limiter à un contrôle plus étroit du phénomène: ils cherchent à promouvoir une image plus positive de la migration et du potentiel qu'elle comporte pour le développement d'un pays. De même, le recrutement de personnes hautement qualifiées peut revêtir un intérêt propre à la migration pour la Suisse. D'autres facettes d'un tel partenariat porteront, comme on l'a dit, sur le pays partenaire lui-même, par exemple en vue d'y combattre la pauvreté et d'y améliorer l'accès à la formation. Cette multiplicité d'objectifs possibles fait que l'on ne constate pas encore de consensus clair au sein de l'administration fédérale à propos des partenariats dans le domaine migratoire. Les priorités varient selon que l'on se concentre sur la migration «indésirable» (portant à renforcer le contrôle) ou sur la migration «désirable» (considérée comme un facteur de développement).

Les pays partenaires ont pour intérêt prioritaire la possibilité offerte à leurs ressortissants d'accéder en toute légalité au marché suisse de l'emploi. Dans son rapport rendu en 2004, le groupe IDAG Migration considère qu'il n'y a pas lieu, dans un proche avenir, de modifier les dispositions de la politique migratoire helvétique. Il convient notamment de maintenir le double système d'admission. L'Accord sur la libre circulation des personnes limite considérablement la marge de manœuvre de la Confédération à l'égard des ressortissants des pays de l'Union européenne (UE); par ailleurs, l'ouverture légale des frontières suisses à des personnes professionnellement peu qualifiées d'Etats tiers ${ }^{9}$ émettrait de mauvais signaux et susciterait des incitations inopportunes ${ }^{10}$. En principe, cette orientation générale ne devrait pas être modifiée non plus pour les partenariats migratoires. Ne sont à l'ordre du jour que des programmes de formation ou de perfectionnement, des séjours de durée limitée pour des stagiaires ou certaines formes particulières du statut de saisonnier, qui pourraient être offerts aux pays partenaires. Toutes ces propositions excluent l'accès durable au marché de l'emploi et donc à de nombreux systèmes de sécurité sociale. Rien ne semble indiquer pour le moment que les partenariats migratoires puissent bouleverser en quoi que ce soit le principe du double système d'admission.

8 S. Castles, «The Factors that Make and Unmake Migration Policies», International Migration Review, $\mathrm{n}^{\circ} 3,2004$, p. 861.

9 Les relations entre la Suisse et les pays de l'UE et de l'Association européenne de libre-échange (AELE) sont réglées par des dispositions particulières. Les partenariats migratoires dont il est question ici concernent exclusivement des Etats tiers.

10 Interdepartementale Arbeitsgruppe, op. cit., pp. 17-18. 


\section{La collaboration interdépartementale}

La collaboration entre départements au sein de l'administration fédérale suisse en matière de politique migratoire est à la fois un préalable et la clé de partenariats réussis dans ce domaine. Il existe depuis plusieurs années différents organes de collaboration interdépartementale pour s'occuper de cette problématique. Tous les départements sont ainsi représentés dans le Groupe de travail interdépartemental pour la migration (GIM), créé en 1998. Une commission du GIM, «Fokus Migration», mise sur pied en 2005 et coprésidée par l'ODM et le DFAE, planifie et coordonne entre autres les stratégies relatives aux partenariats migratoires. Le Groupe directeur interdépartemental d'aide au retour (ILR) constitue l'organe de coordination et de pilotage pour les activités de l'ODM et de la DDC dans le domaine du retour. L'ensemble des prestations de l'ILR conseils en vue du retour, projets en Suisse (formation), aide au retour individuelle et aide structurelle dans le pays de provenance - a été élaboré en 1993, puis institutionnalisé en $1998^{11}$.

Seule cette collaboration interdépartementale peut assurer la cohérence politique de la Suisse à l'égard d'un pays partenaire. Elle accroît en outre les chances d'offrir au pays partenaire des propositions intéressantes à négocier.

Un premier projet pilote de partenariat migratoire est mis en place actuellement avec la partie occidentale des Balkans (Kosovo, Bosnie-et-Herzégovine et Serbie); il est officiellement de la compétence de l'ODM $^{12}$. La condition préalable d'un tel partenariat est un dialogue déjà engagé en la matière, ce qui est le cas en l'occurrence du fait que les Balkans occidentaux constituent depuis le milieu des années 1990 une priorité de la politique étrangère suisse ${ }^{13}$. D'autres partenariats analogues, par exemple avec le Nigeria, sont actuellement à l'étude.

\section{Conclusions}

Les partenariats migratoires ont pour but de gérer les mouvements migratoires de façon à ce que les intérêts de toutes les parties en présence soient traités à égalités de droits. Une bonne collaboration interdépartementale au sein de l'administration fédérale accroît les possibilités de faire des offres plus intéressantes à un pays partenaire et de contribuer ainsi au succès des négociations. En termes de politique étrangère, la Suisse aussi bien que ses interlocuteurs sont limités par leurs législations respectives quant aux concessions qu'ils peuvent faire. C'est notamment le cas pour l'accès légal au marché suisse de l'emploi, que les partenaires revendiquent mais dont la Suisse ne souhaite pas faire un objectif dans un avenir proche. Il semble ainsi à première vue que ces partenariats n'au-

11 Groupe directeur interdépartemental d'aide au retour (ILR), Review der bisherigen Rückkehrhilfeprogramme der ILR: Teil I, Berne, Office fédéral des réfugiés; Direction du développement et de la coopération, 2003.

12 Ordonnance sur l'organisation du Département fédéral de justice et police, <http://www.gesetze.ch/ sr/172.213.1/172.213.1_005.htm> (consulté le 4 avril 2008).

13 Groupe directeur interdépartemental d'aide au retour (ILR), Contribution de l'ILR concernant les partenariats migratoires entre la Suisse et les Balkans occidentaux: Stratégie 2007-2009, Kosovo, Bosnie-et-Herzégovine, Serbie, Berne, Office fédéral des migrations; Direction du développement et de la coopération, 2007. 
ront pas d'incidence majeure sur la politique migratoire poursuivie jusqu'à présent. Mais un second regard permet de réfuter cete observation: alors qu'en 2003 encore on avait l'idée de forcer les pays de provenance à coopérer sous la menace de supprimer les apports financiers à leur développement, la Suisse a reconnu depuis lors qu'elle manquait de puissance pour ce faire. Prendre en compte les intérêts des pays partenaires est le seul moyen d'aboutir à des solutions durables. Autre signe de changement, le débat sur les partenariats migratoires a entraîné une prise de conscience des liens qui existent entre divers aspects de la migration - et l'on aborde ainsi cette problématique complexe de façon plus globale. Cela signifie concrètement que les responsables politiques s'intéressent non seulement aux risques inhérents à la migration, mais aussi aux opportunités qu'elle ouvre.

Il faudra attendre la mise en application concrète de plusieurs partenariats migratoires et examiner la manière dont ceux-ci évolueront pour savoir si cet instrument peut servir à canaliser durablement le phénomène des migrations, s'il est à même de susciter des transformations utiles à l'intérieur des pays concernés et de constituer une véritable valeur ajoutée pour toutes les parties la Suisse, le pays partenaire et les personnes migrantes elles-mêmes.

\section{Bibliographie}

Assemblée fédérale de la Confédération suisse, Loi fédérale sur les étrangers, 2005, <http://www. admin.ch/ch/f/ff/2005/6885.pdf> (consulté le 29 août 2007).

Castles, S., «The Factors that Make and Unmake Migration Policies», International Migration Review, $n^{\circ} 3,2004$.

De Matos, C. und D. Efionayi-Mäder, «Begriffsentstehung, Definition und Ausrichtung von Migrationspartnerschaften in der Schweiz», in Internationale Organisation für Migration (Hg.), Migrationspartnerschaften: Eine Bestandesaufnahme: Darstellung des Begriffs im internationalen und schweizerischen Kontext, Bern, Internationale Organisation für Migration (IOM), 2007.

Groupe directeur interdépartemental d'aide au retour (ILR), Contribution de l'ILR concernant les partenariats migratoires entre la Suisse et les Balkans occidentaux: Stratégie 2007-2009, Kosovo, Bosnieet-Herzégovine, Serbie, Berne, Office fédéral des migrations; Direction du développement et de la coopération, 2007.

Groupe directeur interdépartemental d'aide au retour (ILR), Review der bisherigen Rückkehrhilfeprogramme der IL: Teil I, Berne, Office fédéral des réfugiés; Direction du développement et de la coopération, 2003.

Interdepartementale Arbeitsgruppe «Aussenpolitik im Migrations- und Rückkehrbereich» (IDAG Migration), Schlussbericht der IDAG Migration (résumé disponible en français), Berne, 2004.

Liechti, T., Migrationspolitik: ein neuer Ansatz der schweizerischen Migrationspolitik?, Fribourg, Departement Sozialarbeit und Sozialpolitik, Universität Fribourg, 2007.

Mona, P., «UN High-level Dialogue on International Migration and Development», Going Home: Vierteljährlicher Newsletter der Rückkehrhilfe Kommunikation (RüKo), Bern, 2006.

Nationalrat, Sondersession Mai 2004, Dokumentenzentrale, Bern, 2004, <http://www.parlament.ch/se-nrmai-2004.pdf> (consulté le $1^{\text {er }}$ septembre 2007).

Office fédéral des réfugiés, Institution d'un groupe de travail pour de nouveaux progrès dans le domaine de la migration et des retours, communiqué aux médias, Berne, 2003, <http://www.ejpd.admin.ch/ ejpd/fr/home/dokumentation/mi/2003/2003-04-160.html> (consulté le 16 juillet 2007).

Ordonnance du 17 novembre 1999 sur l'organisation du Département fédéral de justice et police, <http:// www.admin.ch/ch/f/rs/c172_213_1.html> (consulté le 4 avril 2008).

Organisation internationale pour les migrations, Enquête sur les partenariats migratoires, Berne, Organisation internationale pour les migrations (OIM), 2007. 